OPEN ACCESS

Edited by:

Christian Apitz

Ulm University Medical

Center, Germany

Reviewed by:

Ulrich Neudorf

Essen University Hospital, Germany

Jochen Weil,

Technical University Munich, Germany

Taisto Sarkola,

University of Helsinki, Finland

*Correspondence:

Olof Broberg

olof.broberg@med.lu.se

orcid.org/0000-0002-1566-5780

Specialty section:

This article was submitted to

Pediatric Cardiology,

a section of the journal

Frontiers in Pediatrics

Received: 25 August 2021

Accepted: 22 October 2021

Published: 08 December 2021

Citation:

Broberg O, Øra I, Wiebe T, Weismann CG and Liuba P (2021)

Characterization of Cardiac, Vascular, and Metabolic Changes in Young

Childhood Cancer Survivors.

Front. Pediatr. 9:764679.

doi: 10.3389/fped.2021.764679

\section{Characterization of Cardiac, Vascular, and Metabolic Changes in Young Childhood Cancer Survivors}

\author{
Olof Broberg ${ }^{1,2 \star}$, Ingrid Øra ${ }^{2,3}$, Thomas Wiebe ${ }^{3}$, Constance G. Weismann ${ }^{1,2}$ and \\ Petru Liuba ${ }^{1,2}$ \\ ${ }^{1}$ Pediatric Heart Center, Skåne University Hospital, Lund, Sweden, ${ }^{2}$ Clinical Sciences, Department of Pediatrics, Lund \\ University, Lund, Sweden, ${ }^{3}$ Pediatric Oncology, Skåne University Hospital, Lund, Sweden
}

Background: Childhood cancer survivors (CCS) are at an increased risk for cardiovascular diseases (CVD). It was the primary aim of this study to determine different measures of cardiac, carotid, lipid, and apolipoprotein status in young adult CCS and in healthy controls.

Methods: Cardiac and common carotid artery (CCA) structure and function were measured by ultrasonography. Lipids and apolipoproteins were measured in the blood. Peripheral arterial endothelial vasomotor function was assessed by measuring digital reactive hyperemia index (PAT-RHI) using the Endo-PAT 2000.

Results: Fifty-three CCS (20-30 years, 35 men) and 53 sex-matched controls were studied. The CCS cohort was divided by the median dose of anthracyclines into a low anthracycline dose (LAD) group (50-197 mg/m²,$n=26)$ and a high anthracycline dose (HAD) group (200-486 mg/m²,$n=27$ ). Carotid distensibility index (DI) and endothelial function determined by PAT-RHI were both lower in the CCS groups compared with controls $(p<0.05$ and $p=0.02$ ). There was no difference in carotid intima media thickness. Atherogenic apolipoprotein-B (Apo-B) and the ratio between Apo-B and Apoliprotein-A1 (Apo-A1) were higher in the HAD group compared with controls ( $p$ $<0.01$ ). Apo-B/Apo-A1-ratio was over reference limit in $29.6 \%$ of the HAD group, in $15.4 \%$ of LAD group, and in $7.5 \%$ of controls $(p=0.03$ ). Measured lipid markers (low density lipoprotein and total cholesterol and triglycerides) were higher in both CCS groups compared with controls $(\rho<0.05)$. Systolic and diastolic function were measurably decreased in the HAD group, as evidenced by lower EF $(p<0.001)$ and lower é-wave $(p<0.005)$ compared with controls. CCA DI correlated with Apo-B/Apo-A1-ratio and Apo-A1. Follow-up time after treatment correlated with decreased left ventricular ejection fraction $(p=0.001)$.

Conclusion: Young asymptomatic CCS exhibit cardiac, vascular, lipid, and apolipoprotein changes that could account for increased risk for CVD later in life. These findings emphasize the importance of cardiometabolic monitoring even in young CCS.

Keywords: childhood cancer, cardiac function, carotid artery, reactive hyperaemia index, apolipoprotein 


\section{INTRODUCTION}

Cardiovascular disease (CVD) including cardiomyopathy, ischemic heart disease, and cerebrovascular disease amount to the most prevalent non-cancerous causes of death in childhood cancer survivors (CCS) $(1,2)$. The risk for CVD in CCS is at least in part attributed to previous treatment regimens with cardiotoxic anthracyclines (AC) and radiotherapy (3). Recent studies have shown that CCS have increased carotid intima media thickness (CIMT), stiffer arteries, and endothelial dysfunction (4-8). These deviations are now recognized as markers of early atherosclerosis and predict later onset of ventricular dysfunction, stroke, and ischaemic heart disease (9-13). CCS are also at increased risk for the metabolic syndrome, dyslipidaemia, obesity, and hormonal abnormalities (14), which could further contribute to their multifold risk for cardiovascular morbidity and mortality (15).

Previous studies have largely focused on either cardiac or vascular abnormalities, mostly in patients during or soon after treatment or in older CCS. Assessing the early onset of both vascular and cardiac changes may provide more accurate insights with regard to the development of future CVD in CCS, leading to better preventive strategies. Therefore, the aim of this work was to determine different cardiac, carotid, lipid, and apolipoprotein markers for CVD in young Swedish CCS and the associations between these markers. To evaluate this, we performed a crosssectional study on young CCS treated with AC, without any previous or current overt CVD, and compared them with ageand sex-matched healthy controls.

\section{METHODS AND STUDY POPULATION}

We conducted an observational cross-sectional case-control study of cardiovascular status in young adults who survived cancer during childhood after treatment at the Department of Pediatric Oncology of the Skåne University Hospital Lund, Sweden. All data collection of CCS and controls was performed from April 2014 to April 2017. Inclusion criteria were: cancer diagnosis under the age of 18 , treatment with AC among other chemotherapeutic agents, with and without radiotherapy, survival more than 5 years after disease remission, and age 20-30 years at inclusion. Exclusion criteria were: brain tumor diagnosis (these patients are known to have endocrine disorders and motor deficits), previous overt CVD or any cardiovascular complication during cancer treatment, any chronic disease or syndrome, or pregnancy. Informed written consent was obtained from all study participants. The study was approved by the Regional Ethical Committee for Human Research, Lund, Sweden (DNR 2013/205).

Abbreviations: Apo-B, apolipoprotein B; Apo-A1, apolipoprotein A1; AC, anthracyclines; CCA, common carotid artery; CCS, childhood cancer survivors; CVD, cardiovascular disease; DI, distensibility index; HAD, high anthracycline dose; HDL, high density lipoprotein; IVS, interventricular septum; LAD, low anthracycline dose; LDL, low density lipoprotein; LVEF, left ventricular ejection fraction; LVID, left ventricular inner diameter; LVPW, left ventricular posterior wall; PAT-RHI, peripheral arterial tonometry-reactive hyperaemia index; SF, shortening fraction; SI, stiffness index; TG, triglycerides.
Childhood cancer survivors were identified in the population based BORISS registry (16) for childhood malignancies in southern Sweden. One-hundred-fifty-two CCS met the eligibility criteria and received a written invitation to participate. If no answer was received an additional invitation was sent. An equal number of healthy controls with similar sex and age distribution were recruited by written announcements at the Skåne University Hospital area in Lund, Sweden, and these were examined exactly in the same way and during the same time-period as the CCS.

To evaluate vascular and cardiac effects of previous treatment with AC, CCS were divided into two subgroups based on the median cumulative dose of AC: low cumulative AC-dose (LAD) group and high cumulative dose (HAD) group. AC-doses were converted to doxorubicin equivalents using conversion factors: 0.83 for duanorubicine, 0.67 for epirubicine, 5.00 for idarubicine, and 4.00 for mitoxantrone (17). Age at diagnosis and follow-up time were retrieved from the registry.

All study participants completed a questionnaire (18) previously used in our institution regarding current regular use of medications (cardiovascular medicines, statins), tobacco use (type (cigarettes or smokeless tobacco), dose and frequency), level of physical exercise (sports or gym training, hours/week). Systolic and diastolic brachial blood pressure were measured after $15 \mathrm{~min}$ of rest in supine position in the right arm using a calibrated wall-hung aneroid sphygmomanometer at the same time as carotid ultrasonography was performed. Weight and height were measured (using a calibrated scale and a stadiometer). Overweight was defined as BMI $\geq 25$ and $<30 \mathrm{~kg} / \mathrm{m}^{2}$ and obesity was defined as $\mathrm{BMI} \geq 30 \mathrm{~kg} / \mathrm{m}^{2}$.

\section{Laboratory Analyses}

Fasting blood samples were collected to analyze lipid and apolipoproteinmarkers for CVD in plasma and serumas follows; triglycerides (TG, ref. $0.45-2.60 \mathrm{mM}$ ), low-density lipoprotein (LDL, ref. 1.20-4.30 mM), high-density lipoprotein (HDL, ref. women $1.00-2.70 \mathrm{mM}$, men $0.80-2.10 \mathrm{mM}$ ), total cholesterol (ref. 2.90-6.10 mM), creatinine (ref. women $45-90 \mu \mathrm{M}$, men

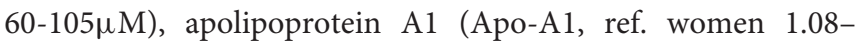
$2.25 \mathrm{~g} / \mathrm{L}$, men $1.04-2.02 \mathrm{~g} / \mathrm{L}$ ), and apolipoprotein B (Apo$\mathrm{B}$, ref. women $0.60-1.17 \mathrm{~g} / \mathrm{L}$, men $0.66-1.33 \mathrm{~g} / \mathrm{L})$. The ApoB/Apo-A1 ratio was calculated (ref. $<0.90$ for men and $<$ 0.80 for women). Estimated glomerular filtration rate (eGFR, ref. $80-125 \mathrm{~mL} / \mathrm{min} / 1.73 \mathrm{~m}^{2}$ ) was estimated from creatinine and cystatine-C (19). All the blood samples were collected and analyzed by standard assays (Roche Diagnostics, Basel, Switzerland). All reference values above are age and sex-specific and were provided by the department of Clinical Chemistry at the Skåne University Hospital.

\section{Echocardiography}

Electrocardiography-gated carotid ultrasonography and echocardiography were performed by a single investigator (OB) according to a standardized protocol, and image acquisition was done according to the American Society of Echocardiography guidelines (20, 21). An echocardiograph (EPIQ-7, Philips Medical Systems, Andover, Massachusetts, USA) equipped with an X5-1 probe (1-5 MHz) was used for echocardiography, 
and a L15-7io (7-15 MHz) linear array transducer for vascular ultrasound was used for carotid ultrasound. The investigator who performed these assessments was blinded to the status of the study participants. All cardiac and carotid measurements were performed offline (QLAB, Philips Healthcare Netherlands) and were averaged over three cardiac cycles.

M-mode measurements of the septal thickness (IVS) and left ventricular posterior wall thickness (LVPW) along with left ventricular internal diameters (LVID) in systole and diastole were obtained in the parasternal view to evaluate cardiac morphology. Left ventricular mass was calculated based on the M-mode measures using the Devereux formula (21). Shortening fraction (SF) was calculated using the M-mode measures. Left ventricular ejection fraction (LVEF) was calculated using the Simpson biplane method using the apical two- and four-chamber views. Mitral valve E- and A-wave were obtained from the apical fourchamber view. To determine the left ventricular ś and é-wave, tissue-Doppler was recorded at mitral annulus septal and lateral wall point (21).

Loops of three to five beats of both right and left common carotid arteries (CCA) in the longitudinal view were obtained using electrocardiography-gated ultrasonography. The far wall of a $1-\mathrm{cm}$ long segment of the proximal CCA was used to measure intima media thickness (CIMT) with a semiautomated tracing algorithm (QLAB, Philips Healthcare Netherlands). The CCA diastolic diameter (CCA Dd) and systolic diameter (CCA Ds) were measured from the leading edge of the near wall (adventia/media border) to the leading edge of the far wall. The distensibility index (DI) and the $\beta$-stiffness index (SI) were calculated using previously described methods $(22,23)$ :

$$
\begin{gathered}
D I\left(\frac{\% \text { diameter change }}{10 m m H g}\right)=\frac{D s-D d}{D d^{*}[S B P-D B P]} * 1000 \\
S I \text { (no unit) }=\ln \left(\frac{S B P}{D B P}\right) /\left(\frac{[D s-D d]}{D d}\right)
\end{gathered}
$$

Inter- and intraobserver variability for the ultrasound measurements were assessed in a subgroup of 23 participants. Interobserver and intraobserver intraclass correlation coefficient (ICC) for all carotid, doppler, and M-mode measurements were $>0.9$. Interobserver ICC for LVEF was 0.84 and 0.90 for intraobserver ICC.

\section{Peripheral Arterial Tonometry-Reactive Hyperaemia Index}

Peripheral vascular endothelial function was assessed by measuring peripheral arterial tonometry-reactive hyperaemia index (PAT-RHI) using the Endo-PAT 2000 (Itamar Medical, Caesarea, Israel). This method has previously been reported to correlate with endothelial function in the coronary circulation (24) and to be predictive for CVD (25). All measurements were done following a standardized procedure specified by the manufacturer (www.itamar-medical.com). After $5 \mathrm{~min}$ of tracing bilateral baseline index-finger oscillations of blood flow, a blood pressure cuff was inflated to occlude blood flow into the nondominant arm for $5 \mathrm{~min}$. Following pressure release, tracings were obtained for another $5 \mathrm{~min}$. A computerized software with a proprietary algorithm automatically calculated the reactive hyperaemia index from the fold increase in the pulse wave amplitude relative to baseline. The reference limit for PAT-RHI was set at $>1.67$, as specified by the manufacturer.

\section{Statistical Analyses}

Statistical analyses were performed with SPSS software (IBM, version 27; SPSS, Chicago, Illinois, USA). Results were reported as means (adjusted and unadjusted) and standard deviations (SD) or numbers (proportions and percentages). Based on the histogram inspection, summary statistics of the software (providing information on means and standard deviations and on medians and quantiles), and given skewness $>-1$ but $<1$, we assumed variables to be approximately normally distributed.

For baseline characteristics, continuous variables were compared between groups using ANOVA and Student $T$-test as appropriate. For dichotomous variables the Chi-square test was used. For group comparisons of outcome variables (DI/SI, CIMT, PAT-RHI, cardiac diastolic and systolic function, and lipids and apolipoproteins) ANCOVA was used. Unbalanced baseline variables were adjusted for. Blood pressure was adjusted for height. In a subgroup analysis adjusting for treatment variables (follow-up time after treatment completion, cranial and mediastinal radiotherapy) only the LAD and the HAD groups were compared. For carotid measures, CCS treated with cranial radiotherapy was compared with CCS without this treatment and results were adjusted for the cumulative AC dose. Post-hoc tests were performed using the Bonferroni method for three-group comparisons and LSD for subgroup analysis of only CCS. Partial correlations adjusted for age and sex were performed within the CCS group. For ANCOVA, means and adjusted means were reported. A two-sided $p$-value below 0.05 was considered statistically significant.

\section{RESULTS}

\section{Characteristics of the Study Participants}

Description of the study cohort including distribution of cancer diagnosis is shown in Table 1. Fifty-three CCS and an equal number of controls (mean age $25.3 \pm 2.4$ and $24 \pm 2.4$ years, respectively), were studied. The median AC dose of CCS was $197.0 \mathrm{mg} / \mathrm{m}^{2}$. As described in the Methods, based by the median AC dose, the CCS cohort was divided into two groups: low AC dose (LAD; $n=26$ ) and high AC dose (HAD; $n=27)$. Among CCS, leukemia was the most common cancer diagnosis $(n=$ 23 of whom three had been treated for acute myeloid leukemia and 20 for acute lymphoblastic leukemia). Four CCS had been treated for rhabdomyosarcoma, one for Ewing's sarcoma, one for osteosarcoma, five for Wilms's tumor, 11 for Hodgkin's disease, and eight for non-Hodgkin's lymphoma.

In addition to AC, 24 CCS (11 in the LAD group and 13 in the HAD group) had radiotherapy as follows: mediastinal radiotherapy in 10 CCS (all with lymphoma, of whom eight 
TABLE 1 | Characteristics of the study cohort divided in subjects with low (LAD) or high (HAD) cumulative dose of anthracyclines.

\begin{tabular}{|c|c|c|c|}
\hline Variables & Controls $(n=53)$ & LAD $(n=26)$ & HAD $(n=27)$ \\
\hline Age, years (SD) & $24.4(2.4)$ & $25.0(2.4)$ & $25.6(2.5)$ \\
\hline Male sex, $\mathrm{n}$ & 35 (66.0\%) & $15(57.7 \%)$ & $17(63.0 \%)$ \\
\hline Height, cm (SD) & $179.1(8.7)$ & $178.0(10.1)$ & $171.8(9.8)^{\star \star}$ \\
\hline Weight, kg (SD) & $79.2(15.3)$ & $78.2(14.4)$ & $71.6(13.3)$ \\
\hline $\mathrm{BMI}, \mathrm{kg} / \mathrm{m}^{2}$ (SD) & $24.60(3.79)$ & $24.64(3.53)$ & $24.17(3.58)$ \\
\hline SBP, mmHg, (SD) & $118.8(11.6)$ & $118.2(10.1)$ & $119.1(12.4)$ \\
\hline DBP, mmHg, (SD) & $73.4(5.9)$ & 75.7 (8.3) & $76.3(8.8)$ \\
\hline Hypertension, $\mathrm{n}$ & $1(1.9 \%)$ & $2(7.7 \%)$ & $2(7.4 \%)$ \\
\hline Resting HR, beats/min, (SD) & $66.2(11.3)$ & $70.0(11.2)$ & $74.1(11.0)^{\star}$ \\
\hline Exercise (hours/week), (SD) & $4.4(2.9)$ & $4.6(6.5)$ & $4.2(2.2)$ \\
\hline \multicolumn{4}{|l|}{ Cancer Diagnosis } \\
\hline Leukaemia, n & & $5(19.2 \%)$ & $18(66.7 \%)$ \\
\hline Hodgkin lymphoma, n & & $10(38.5 \%)$ & $1(3.70 \%)$ \\
\hline Non-Hodgkin lymphoma, n & & 7 (26.9\%) & $1(3.70 \%)$ \\
\hline Sarcoma, $\mathrm{n}$ & & 0 & $6(22.2 \%)$ \\
\hline Wilms tumor, n & & $4(15.4 \%)$ & $1(3.7 \%)$ \\
\hline Age at diagnosis, y (SD) & & $10.8(5.6)$ & $6.0(4.5)^{\star}$ \\
\hline Follow-up time, y (SD) & & $13.4(5.4)$ & $18.0(5.2)^{*}$ \\
\hline Cumulative $\mathrm{AC}, \mathrm{mg} / \mathrm{m}^{2}$ (SD) & & $143.6(33.1)$ & $277.3(84.9)^{\star}$ \\
\hline Radiotherapy, n & & $11(42.3 \%)$ & $13(48.1 \%)$ \\
\hline Mediastinal radiotherapy, $\mathrm{n}$ & & $8(30.8 \%)$ & $2(7.4 \%)$ \\
\hline
\end{tabular}

Values are presented as mean and SD or as number and \%. p-values were calculated by one-way analysis of variance (ANOVA); intergroup differences were calculated by the Bonferroni post-hoc test with correction for multiple comparisons, standard T-test or with chi-square test: * $p<0.05$ vs controls or LAD vs HAD group; ** $p<0.005$ vs controls. LAD, low anthracycline dose; HAD, Iow anthracycline dose; BSA, body surface area; BMI, body mass index; SBP, systolic blood pressure; DBP, diastolic blood pressure; HR, heart rate, AC, anthracycline.

were in the LAD group and two in the HAD group), cranial radiotherapy in eight (all with leukemia in the HAD group), and radiotherapy to other organs in six (three in each group). The mean follow-up time after the end of cancer treatment in the CCS cohort was $15.8( \pm 5.8)$ years. The LAD group was older at the time of diagnosis and had a shorter follow-up compared with the $\operatorname{HAD}$ group ( $p=0.001$ and $p=0.003$, respectively).

The HAD group was shorter compared with the control group $(p=0.004)$ and had a higher resting heart rate $(p=0.010)$. Blood pressure was similar in CCS compared with controls. There were five users of smokeless tobacco in the control group and five and four in the LAD and HAD groups, respectively. The control group had four cigarette users (two daily users and two occasional or previous users). The LAD-group had one daily user and the HAD group had two occasional or previous users. There was no difference in tobacco use between controls and CCS groups. None of the participants were on cardiovascular medications. Exercise (hours/week) was similar between controls and CCS.

\section{Apolipoproteins and Lipid Biomarkers}

Results of group comparisons between controls and CCS groups of lipid biomarkers and apolipoproteins are outlined in Table 2. Compared with controls, markers for atherosclerosis, Apo-B and the Apo-B/Apo-A1-ratio, were higher in the HAD group ( $p=0.006$ and $p=0.011$, respectively). Apo-B/Apo-A1 ratio exceeded the upper reference range for age and sex in $7.5 \%$ of controls compared with $15.4 \%$ in the LAD group and $29.6 \%$ in the HAD group $(p=0.030)$. Apo-A1 and HDL were similar between controls and CCS groups. Compared with controls LDL, total cholesterol, and TG were higher in the HAD group ( $p$ $<0.005$ ). Following adjustment for radiotherapy and followup time, cholesterol and Apo-B were higher in the HAD group when compared to the LAD group $(p=0.040$ and $p=$ 0.041 , respectively).

In order to exclude a possible confounding effect of overweight and obesity on Apo-B and the Apo-B/Apo-A1 ratio, a separate analysis of CCS $(n=29)$ and controls $(n=32)$ with a normal weight $\left(\mathrm{BMI}<25 \mathrm{~kg} / \mathrm{m}^{2}\right.$ ) was done: CCS had higher 
TABLE 2 | Different biomarkers for lipid status in childhood cancer survivors with low (LAD) or high (HAD) cumulative dose of anthracyclines.

\begin{tabular}{|c|c|c|c|c|c|}
\hline \multirow[t]{2}{*}{ Variables } & \multirow[t]{2}{*}{ Controls } & \multicolumn{2}{|c|}{ LAD $(n=26)$} & \multicolumn{2}{|c|}{ HAD $(n=27)$} \\
\hline & & \multicolumn{2}{|c|}{ Subgroup analysis ${ }^{a}$} & \multicolumn{2}{|c|}{ Subgroup analysis ${ }^{a}$} \\
\hline LDL (mM) & $2.22(0.77)$ & $2.64(0.69)^{\star}$ & & $3.06(0.77)^{\star \star}$ & \\
\hline Adjusted Means & $2.25(0.77)$ & $2.63(0.74)$ & $2.61(0.83)$ & $2.69(0.78)$ & $3.05(0.83)$ \\
\hline HDL (mM) & $1.40(0.32)$ & $1.36(0.30)$ & & $1.29(0.34)$ & \\
\hline Adjusted Means & $1.41(0.33)$ & $1.37(0.32)$ & $1.34(0.36)$ & $1.28(0.34)$ & $1.33(0.36)$ \\
\hline $\mathrm{TG}(\mathrm{mM})$ & $0.79(0.35)$ & $1.01(0.58)^{\star}$ & & $1.37(0.93)^{\star \star}$ & \\
\hline Adjusted Means & $0.81(0.58)$ & $1.00(0.59)$ & $1.04(0.85)$ & $1.33(0.62)$ & $1.32(0.85)$ \\
\hline Cholesterol $^{\mathrm{b}}$ (mM) & $3.84(0.82)$ & $4.32(0.76)^{\star}$ & & $4.82(0.88)^{\star \star}$ & \\
\hline Adjusted Means & $3.89(0.83)$ & $4.31(0.78)$ & $4.29(0.90)$ & $4.71(0.85)$ & $4.85(0.92)^{b}$ \\
\hline Apo-A1 (g/L) & $1.42(0.22)$ & $1.57(0.40)$ & & $1.44(0.27)$ & \\
\hline Adjusted Means & $1.44(0.24)$ & $1.45(0.23)$ & $1.44(0.28)$ & $1.43(0.24)$ & $1.48(0.29)$ \\
\hline Apo-B (g/L) & $0.72(0.20)$ & $0.80(0.19)$ & & $0.92(0.21)^{\star \star}$ & \\
\hline Adjusted Means & $0.73(0.20)$ & $0.80(0.19)$ & $0.79(0.22)$ & $0.90(0.20)$ & $0.93(0.21)^{b}$ \\
\hline Apo-B/Apo-A1 ratio & $0.52(0.16)$ & $0.56(0.14)$ & & $0.65(0.19)^{\star \star}$ & \\
\hline Adjusted Means & $0.52(0.17)$ & $0.56(0.16)$ & $0.56(0.19)$ & $0.65(0.17)$ & $0.65(0.18)$ \\
\hline
\end{tabular}

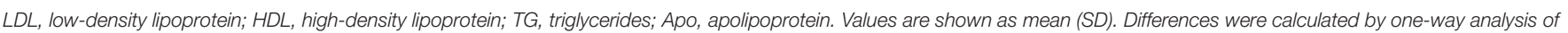

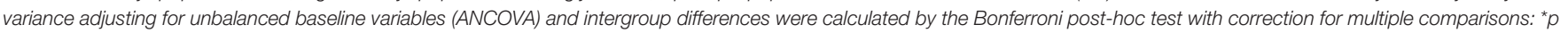

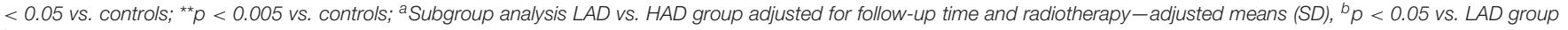

${ }^{b} 1$ sample result for cholesterol missing in the HAD group. Italic values are subgroup analysis.

Apo-B of $0.85,95 \%$ CI [0.78-0.92] vs $0.69,95 \%$ CI [0.62-0.76], $p$ $=0.001$ and Apo-B/Apo-A1-ratio of $0.58,95 \% \mathrm{CI}[0.52-0.64]$ vs $0.48,95 \%$ CI $[0.42-0.54], p=0.016$.

\section{Carotid Measurements and PAT-RHI}

These data are outlined in Table 3. CIMT was not different between controls and CCS groups $(p=0.90)$. The mean DI of the right and left CCA was lower in both LAD and HAD groups compared with controls ( $p<0.001$ and $p=0.018$, respectively). The mean SI of the right and left CCA was higher in the LAD and HAD groups compared with controls $(p=0.041$ and $p<$ 0.001 , respectively). Further, mean DI was lower, and mean SI was higher in the HAD group compared with the LAD group ( $p=$ 0.040 and $p=0.002$, respectively). After adjusting for follow-up time and cranial and mediastinal radiotherapy, the HAD group displayed lower DI and higher SI compared to the LAD group ( $p$ $=0.007$ and $p=0.020$, respectively).

When comparing CCS with and without cranial radiotherapy (Table 4), SI of the left CCA was higher in those with cranial radiotherapy $(p=0.011)$ after adjusting for the total AC dose.

Peripheral arterial tonometry-reactive hyperaemia index was significantly lower in the whole CCS cohort compared with controls (CCS; 1.94, 95\% CI 1.77-2.12, controls; 2.23, 95\% CI $2.06-2.40, p=0.013)$. Likewise, the proportion of study participants with an abnormal PAT-RHI defined as $<1.67$ was significantly higher in the CCS cohort when compared with the controls ( $n=19$ of CCS vs. nine of controls, $p=0.046$ ). The LAD group, but not the HAD group, had lower PAT-RHI compared with controls $(p=0.017)$ and there was no difference between the LAD and the HAD group $(p=0.36)$, as shown in Table 3. In the subgroup analysis, controlling for follow-up time and radiotherapy, comparing the $\mathrm{LAD}$ and the HAD groups, there was no difference between the groups for PAT-RHI $(p=0.47)$.

\section{Echocardiography}

Group comparisons are outlined in Table 5. Both CCS groups had lower cardiac mass compared with controls $(p=0.011$ and $p=0.041$, respectively). Compared with controls and the LAD group, systolic LVID was larger in the HAD group $(p=0.001$ and $p=0.014$, respectively). Systolic and diastolic LVPW were smaller in both the CCS groups when compared with controls $(p$ $<0.05$ for diastolic LVPW and $p<0.005$ for diastolic LVPW). LV systolic function (LVEF) was lower in the HAD group compared with controls and the LAD group $(p<0.001$ and $p=0.020$, respectively). Six CCS in the HAD group had LVEF $<55 \%$ (49$54.2 \%$ ) compared with none in the LAD group and one among controls (LVEF 54.9\%).

Tissue Doppler mean septal and lateral wall systolic 's-wave was lower in both the CCS groups compared with controls ( $p$ $=0.002$ for HAD group and $p=0.029$ for LAD group). Mean tissue Doppler é-wave (as a measurement of diastolic function) was lower in the CCS groups compared with controls: $(p=0.001$ for LAD group and $p<0.001$ for HAD group). In the subgroup analysis comparing CCS groups, adjusting for follow-up time and radiotherapy mean é-wave was lower in the HAD group compared with the LAD group $(p=0.049)$.

\section{Correlations of Cardiovascular Outcome Variables Within the CCS Groups}

Correlation analyses between total AC dose and outcome variables corrected for age and sex revealed moderate negative correlations with LVEF $(r=-0.46, p<0.001)$, SF $(r=-0.43, p$ $=0.002$ ), and with mean right and left CCA DI, a weak-moderate correlation $(r=-0.30, p=0.046)$. Cranial radiotherapy was correlated only with mean right and left CCA DI and SI ( $r=$ $-0.31, p=0.038$ for DI and $r=0.31, p=0.034$ for SI). There were no significant correlations of CIMT, PAT-RHI and lipids 
TABLE 3 | Vascular outcomes in controls and childhood cancer survivors with low (LAD) or high (HAD) cumulative dose of anthracyclines.

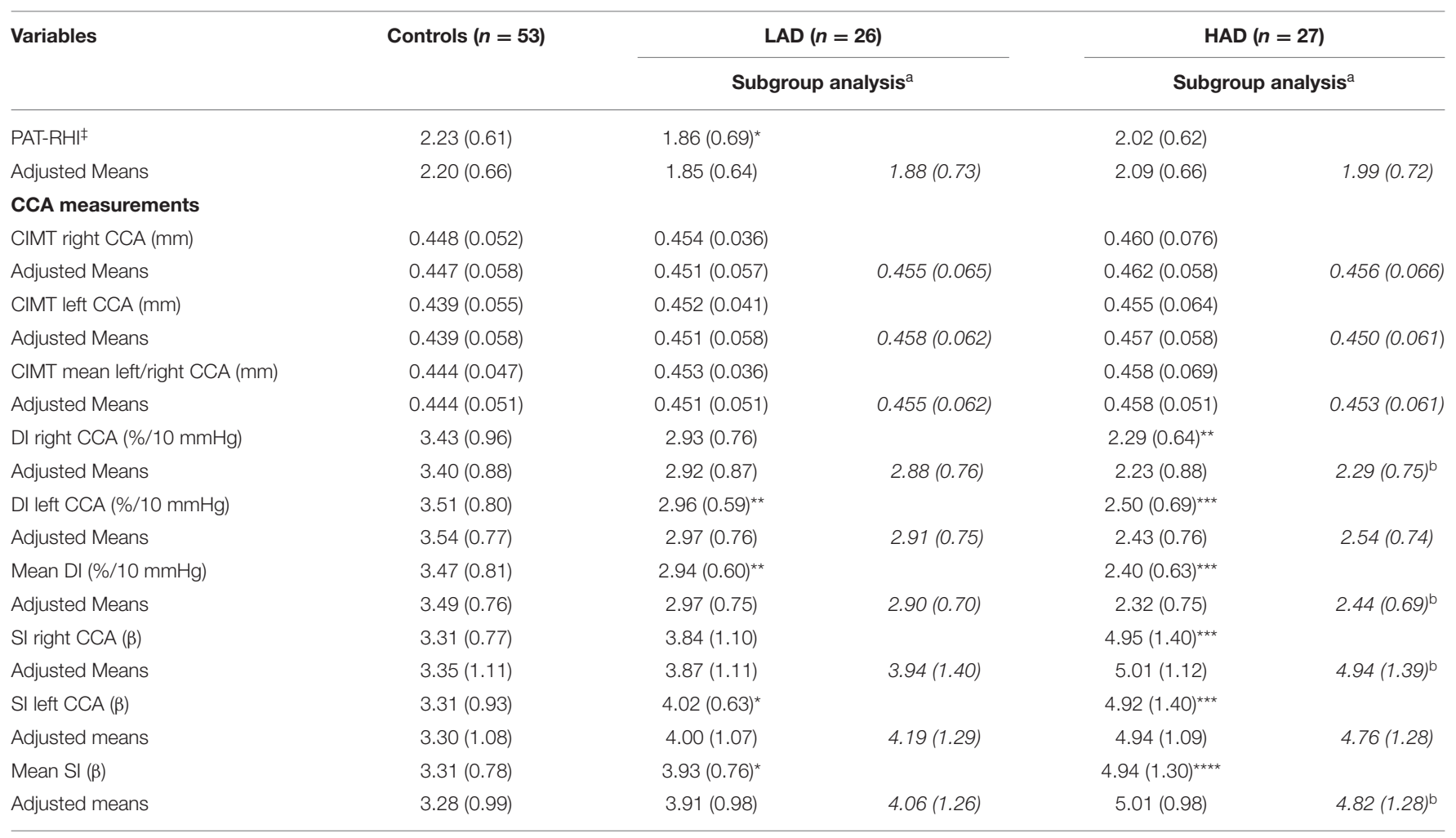

PAT-RHI, reactive hyperaemia index; CCA, common carotid artery; CIMT, carotid intima media thickness; DI, distensibility index; SI, stiffness index. Values are shown as mean (SD). Group differences were calculated by one-way analysis of covariance adjusting for heart rate and height (ANCOVA). Intergroup differences were calculated by the Bonferroni post-hoc test with correction for multiple comparisons; ${ }^{*} p<0.05$ vs. control, ${ }^{* *} p<0.005$ vs. control, ${ }^{* * *} p<0.05$ vs. LAD group and $p<0.005$ vs. controls, ${ }^{* * * *} p<0.005$ vs. controls and LAD group, a Subgroup analysis LAD vs. HAD group adjusted for follow-up time and radiotherapy-adjusted means (SD), ${ }^{b} p<0.05$ vs. LAD group. ${ }^{\ddagger}$, missing data for 1 control and 2 in the $L A D$ group. Italic values are subgroup analysis.

TABLE 4 | Carotid measures in CCS treated with cranial radiotherapy.

\begin{tabular}{|c|c|c|c|}
\hline Variables & $\operatorname{ccs}(N=45)$ & Cranial RT $(N=8)$ & $P$-value \\
\hline CIMT right CCA $(\mathrm{mm})$ & $0.455(0.063)$ & $0.455(0.034)$ & 0.960 \\
\hline Adjusted means & $0.457(0.057)$ & $0.448(0.058)$ & 0.696 \\
\hline CIMT left CCA (mm) & $0.455(0.057)$ & $0.445(0.035)$ & 0.617 \\
\hline Adjusted means & $0.457(0.051)$ & $0.437(0.054)$ & 0.331 \\
\hline CIMT mean left/right CCA (mm) & $0.454(0.058)$ & $0.450(0.026)$ & 0.813 \\
\hline Adjusted Means & $2.64(0.71)$ & $2.19(0.74)$ & 0.123 \\
\hline DI left CCA (\%/10 mmHg) & $2.82(0.66)$ & $2.24(0.60)$ & 0.027 \\
\hline Adjusted means & $2.80(0.64)$ & $2.32(0.65)$ & 0.060 \\
\hline Mean DI (\%/10 mmHg) & $2.75(0.66)$ & $2.18(0.51)$ & 0.023 \\
\hline Adjusted means & $2.74(0.63)$ & $2.25(0.63)$ & 0.054 \\
\hline Adjusted means & $4.29(1.11)$ & $5.47(1.13)$ & 0.011 \\
\hline Mean SI ( $\beta$ ) & $4.27(1.02)$ & $5.36(1.55)$ & 0.121 \\
\hline Adjusted means & $4.34(1.11)$ & $5.27(1.13)$ & 0.099 \\
\hline
\end{tabular}

CCA, common carotid artery; CIMT, carotid intima media thickness; DI, distensibility index; SI, stiffness index; RT, radiotherapy. Values are shown as mean (SD). Group differences were calculated by one-way analysis of covariance adjusting for mean anthracycline dose (ANCOVA). 
TABLE 5 | Cardiac outcomes in controls and childhood cancer survivors with low (LAD) or high (HAD) cumulative dose of anthracyclines.

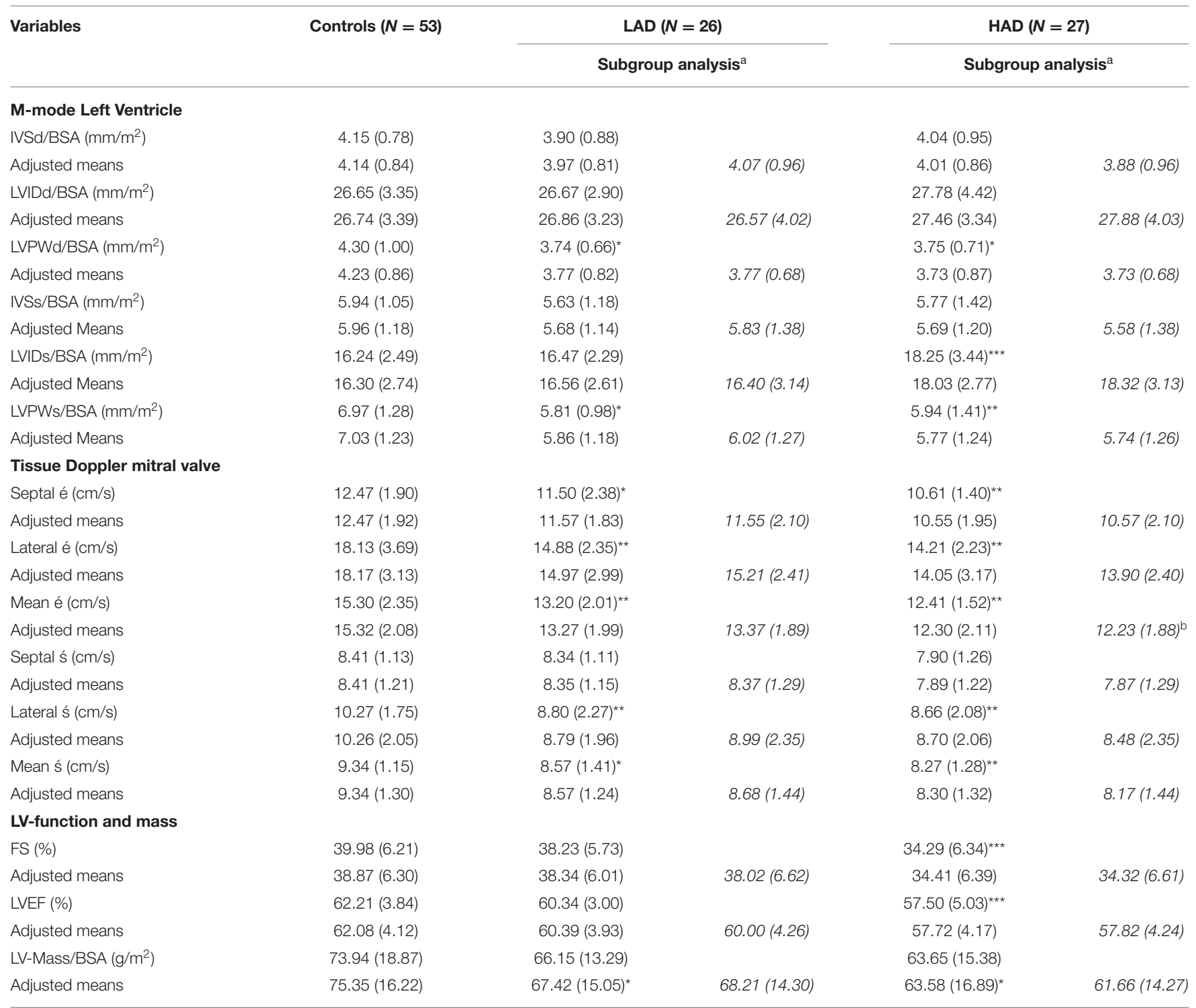

BSA, body surface area; IVSd, septal diastolic diameter; IVSs, septal systolic diameter; LVIDd, left ventricular diastolic inner diameter; LVIDs, left ventricular systolic inner diameter; LVPWd, left ventricular posterior wall diastolic diameter; LVPWs, left ventricular posterior wall systolic diameter; SF, shortening fraction; LVEF, left ventricular ejection fraction calculated by Simpson method; LV-mass, left ventricular mass. Values are shown as mean (SD). Group differences were calculated by one-way analysis of variance adjusting for heartrate and height (ANCOVA). Intergroup differences were calculated by the Bonferroni post-hoc test with correction for multiple comparisons; ${ }^{*} p<0.05 \mathrm{vs}$. control, ${ }^{* *} p<0.005 \mathrm{vs}$. control, ${ }^{* * *} p<$ 0.05 vs controls and LAD group, a Subgroup analysis $L A D$ vs. HAD group adjusted for follow-up time and radiotherapy-adjusted means (SD), ${ }^{b} p<0.05$ vs. $L A D$ group. Italic values are subgroup analysis.

and apolipoproteins regarding $\mathrm{AC}$ dose, cranial nor mediastinal radiotherapy and follow-up time (data not shown). Follow-up time after cancer treatment was associated with LVEF only $(r=$ $-0.47, p=0.001$, Figure 1).

When focusing on correlations between structural, functional, and biochemical outcome variables, mean right and left CCA DI and SI were correlated with Apo-A1 ( $r=0.41, p=0.005$ for DI, Figure $2 \mathrm{~A}$ and $r=-0.32, p=0.030$ for SI), whereas Apo-B/ApoA1 ratio met statistical significance only in correlation with DI ( $r=-0.34, p=0.020$, Figure 2B). PAT-RHI and CIMT did not correlate with any of the lipid or apolipoprotein markers.

\section{DISCUSSION}

Improvement of long-term follow up of CCS is mandatory to further lower the risk for cardiovascular events in adult life. The findings of this study demonstrate that subclinical cardiac, vascular, and lipid and apolipoprotein disorders are present in CCS already at a young adult age. More specifically, cardiac systolic and diastolic function and the carotid artery's elasticity are affected in CCS with a higher burden of cancer treatment, and the latter correlates with apolipoprotein abnormalities. These ultrasonographical and lipid and apolipoprotein markers 


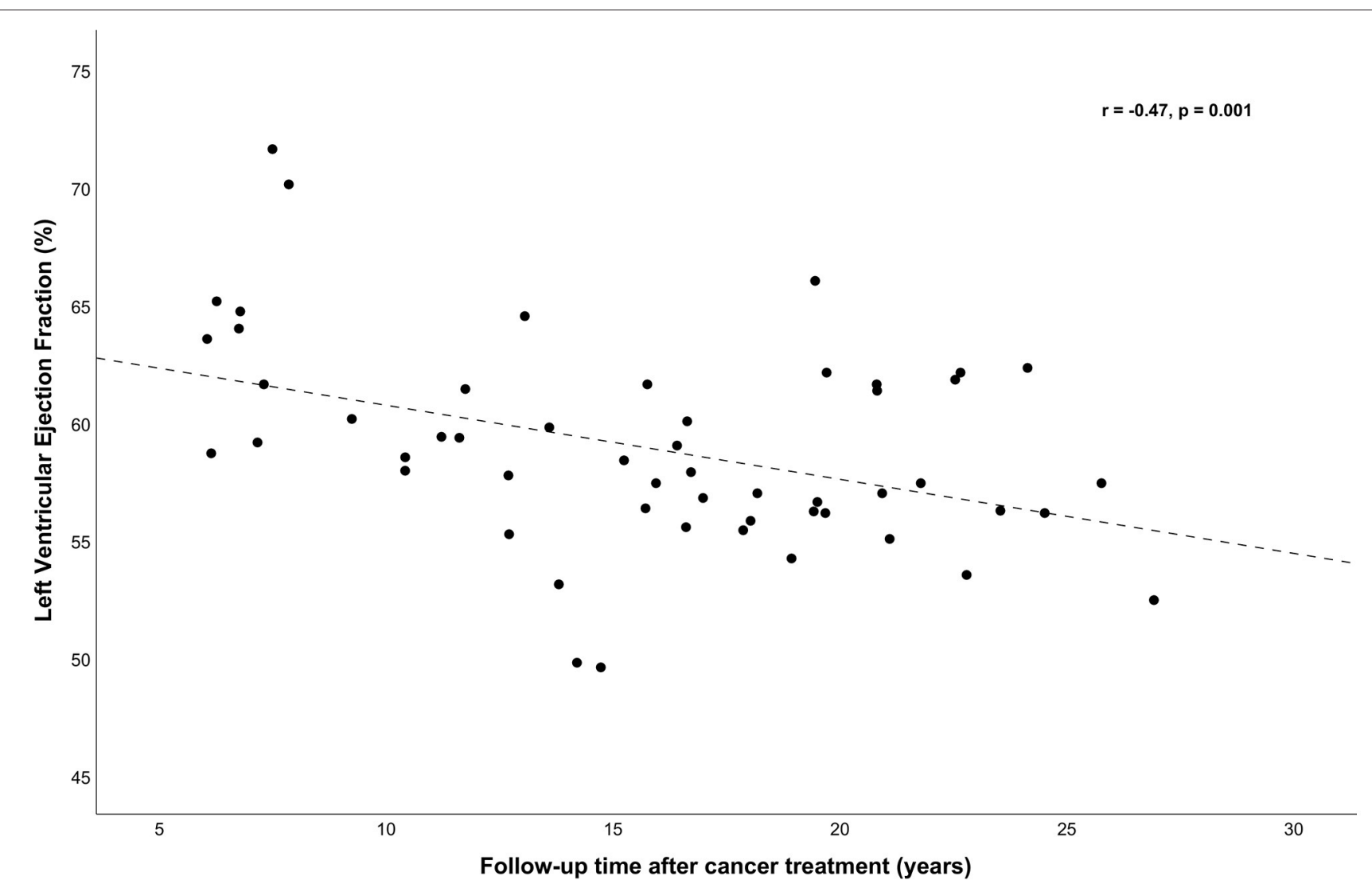

FIGURE 1 | Left ventricular ejection fraction (LVEF, \%) was correlated (adjusted for sex and age) with follow-up time $(r=-0.47, p=0.001)$. With simple linear regression a significant regression equation was found $\left[F_{(1,51)}=10.65, p=0.002\right]$, with an $r^{2}=0.173$. The predicted LVEF (\%) was $63.86-0.315$ (years of follow-up time after cancer treatment).
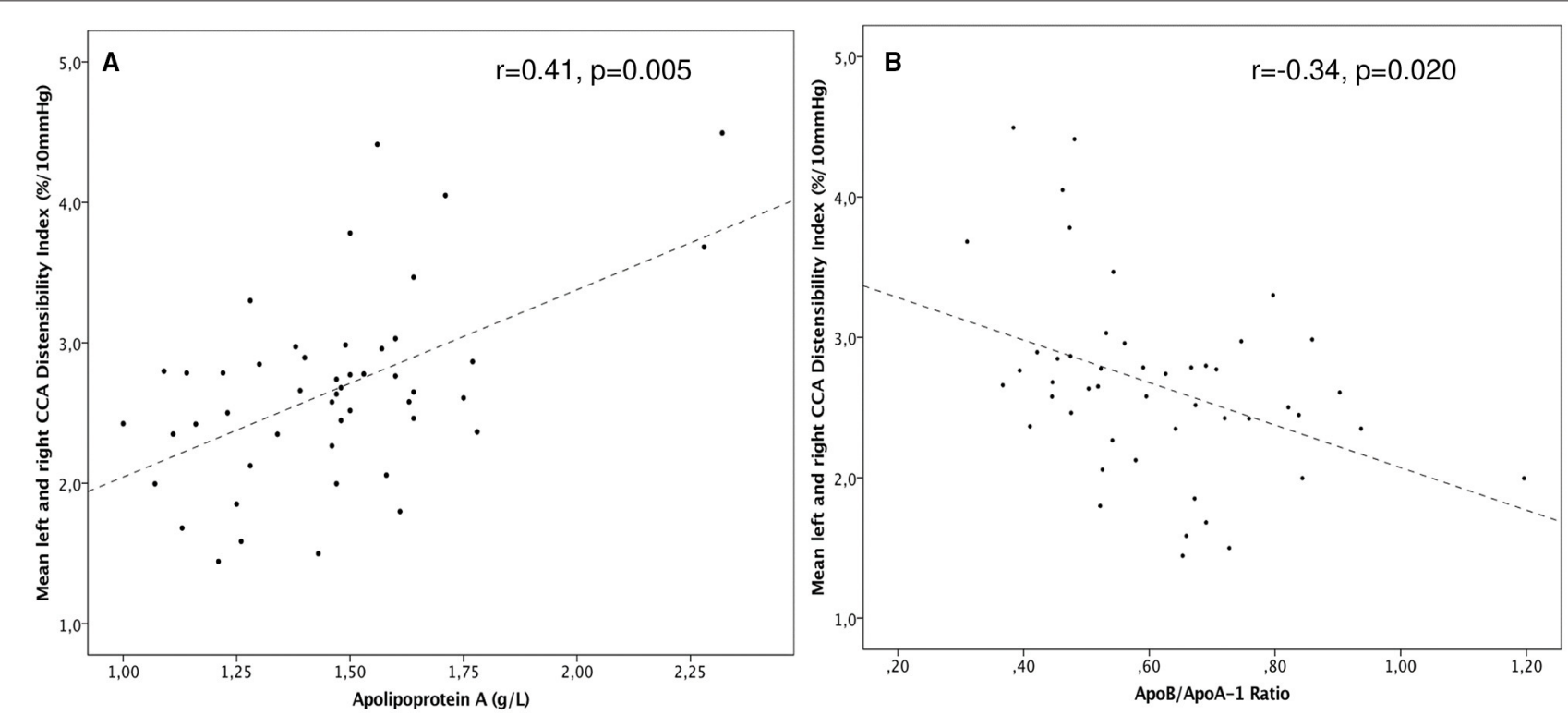

FIGURE 2 | (A) Scatterplot of common carotid distensibility (CCA DI) among CCS was positively correlated with Apo-A $(r=0.41, p=0.005)$. (B) DI vs. the Apo-B/Apo-A1-ratio among CCS. A higher ratio depicts lower DI $(r=-0.34 p=0.020)$. 
are known risk-factors for future CVD in different patient populations (25-29), but how they predict CVD in CCS remains elusive.

\section{Carotid and Cardiac Markers}

Carotid stiffness, a surrogate non-invasive marker for atherosclerosis, appears to be an independent marker for CVD and mortality $(28,29)$ and has been suggested to predict risk for stroke in older CCS (>40 years) (12). In the current study, CCS with a higher AC dose (HAD group) had the most increased carotid stiffness. Similar results were reported in a previous work of CCS (4), where the aortic stiffness was worse in CCS treated with AC as compared with healthy controls. Moreover, in a large study of 852 CCS by Arnold et al. (30), arterial SI was markedly higher than in healthy controls with more pronounced differences with increasing age even among those without hypertension. Carotid stiffness (expressed by DI and SI) in the current study correlated with cranial radiotherapy, indicating that both cranial radiotherapy and AC might account for the observed changes in carotid function. In young cancer survivors after childhood stem cell transplantation, radiotherapy was also reported to be associated with increased carotid stiffness and carotid plaques despite the use of protective shielding of the carotid area (31) suggesting a systemic effect. Radiotherapy in higher doses to the carotid area is known to cause atherosclerosis $(12,31)$.

In the present study, the subclinical changes in the cardiac function (both systolic and diastolic) and in carotid elasticity in CCS showed no correlation with each other. Theoretically, the increased afterload caused by impaired carotid elasticity could affect left ventricular diastolic parameters. This has been described in hypertensive patients in whom arterial stiffening was associated with left ventricular hypertrophy and impaired diastolic function (32). In young CCS the relationship between arterial stiffness and left ventricular diastolic dysfunction might be less obvious since $\mathrm{AC}$ and radiotherapy damage both vascular endothelium and cardiac myocytes and the "normal" timing of events could thus be disrupted. CCS are different from hypertensive patients, as that they do not have left ventricular hypertrophy but instead thinner cardiac walls and decreased left ventricular mass due to cardiomyocyte apoptosis (33). The expected alterations in left ventricular function due to stiffened vasculature in CCS could also require more sensitive methods such as strain rate imaging (34). The younger age of CCS in our study could be important too, since subtle left ventricular structural changes due to arterial stiffness may not be measurable in younger individuals (35).

These effects on the vasculature by cardiotoxic AC can be explained by the putative endothelial toxicity of $\mathrm{AC}$ through oxidative stress and direct DNA-damage acting on vascular endothelial cells, leading to apoptosis and endothelial dysfunction (36). It has also been reported that AC leads to decreased elastin and increased collagen content, suggesting a possible structural derangement caused by AC (37). Like AC, radiotherapy induces oxidative stress (38) leading to inflammation and fibrosis that acts locally in the irradiated area (39). But also, nearby or systemically through the so-called "non-targeted-effects" $(40,41)$ which could in part explain why cranial radiotherapy in the current study correlated with carotid elasticity. However, to separate the individual effects of AC and radiotherapy is complex. The HAD group had higher AC doses and cranial radiotherapy as well as a longer follow-up time and the effects are probably additive (42). There are also other factors in CCS such as previous infections and severity of disease that could affect the cardiovascular system as it has been reported that CCS without chemotherapy or radiotherapy have cardiovascular abnormalities when compared with controls (43). Furthermore, mediastinal radiotherapy in combination with AC increases the risk for CVD substantially $(3,44)$. We could not confirm this in the present study, probably due to relatively short follow-up time (45).

\section{Endothelial Function Assessed With PAT-RHI}

In the present study, endothelial vasomotor function assessed with PAT-RHI was decreased in CCS but no correlation with carotid stiffness nor cardiac function was observed. PAT-RHI has been reported to correlate with endothelial vasomotor response in the coronary circulation (24) and to predict cardiovascular outcome in individuals with CVD risk factors $(25,46)$. PATRHI has been widely studied in other patient cohorts but to our knowledge only two previous studies assessed PAT-RHI in CCS: one in young acute lymphoblastic leukemia survivors (47) and the other in young Hodgkin lymphoma survivors (48). Interestingly, in our study, the LAD group, but not the HAD group, had lower PAT-RHI than controls. Mediastinal radiotherapy was more frequent in the LAD group in the current study while in patients with Hodgkin lymphoma in the previous study (48) mediastinal radiotherapy in conjunction with chemotherapy was associated with a lower PAT-RHI score. The underlying mechanism is unclear, but one explanation could reside in the systemic effects on the endothelial cells of radiotherapy and $\mathrm{AC}(38,39)$. However, despite the growing data that PAT-RHI can predict cardiovascular morbidity, its potential benefit in general population remains debatable and results should be interpreted with caution (49).

\section{Apolipoproteins and Lipids}

In the INTERHEART study of 12,461 individuals with myocardial infarction, an increased Apo-B/Apo-A1 ratio was shown to be superior to any lipid variable in estimating the risk for myocardial infarction across ethnic groups, sexes, and at all ages (26). Likewise, reported in that study, for every increase in Apo-A1 by 1 SD, cardiovascular risk decreased by $33 \%$ compared to $15 \%$ for a similar increase in HDL. In the present study, Apo-A1 and the Apo-B/Apo-A1 ratio among CCS were associated with better- and worse carotid elasticity respectively. Apo-A1, along with HDL, is well-known for its vasoprotective effects, being involved in the "reverse cholesterol transport" from the periphery to the liver and in arterial plaque inhibition in part via antiinflammatory effects (50). Apo-Al is the major protein component of HDL and has been suggested to protect the cardiovascular system against AC toxicity via cytoprotective effects and cardiac "sparing" in delivery of AC 
(51). Whether Apo-A1 might be the driving mechanism of the effect observed herein needs to be assessed in future studies.

Apo-B, suggested to be the basic unit of injury to the arterial wall (52), was higher in the HAD group and in conjunction with this nearly $30 \%$ of CCS in the HAD group had Apo-B/ApoA1-ratio over the upper reference limit, with lower number in the LAD group (15\%) but still higher compared with controls (7\%). This difference could be explained by cranial radiotherapy which was used in $31 \%$ of the HAD group compared to none in the LAD group. Cranial radiation, which was used only the HAD group, has been linked to neuroendocrine dysfunction and growth hormone deficiency that can cause dyslipidemia $(14,53,54)$. Cranial radiotherapy was also correlated with impaired carotid elasticity, and this correlation could be due to the above-mentioned systemic effects of radiotherapy including inflammation and neuroendocrine dysfunction (14, 41, 53, 54). Importantly, normal-weight CCS had higher values of analysed apolipoprotein biomarkers. Based on this observation, we propose that lipid and apolipoprotein screening should be added to the current monitoring of CCS even in those without other signs of metabolic syndrome.

\section{Limitations}

The cross-sectional design and the relatively small number of CCS is an important limitation. Given the cohort size, we did not use the three-group classification (in example: low risk $<100 \mathrm{mg} / \mathrm{m}^{2}$, intermediate risk $100-250 \mathrm{mg} / \mathrm{m}^{2}$, and high risk $>250 \mathrm{mg} / \mathrm{m}^{2}$ ) commonly used in cardiovascular followup of CCS (44). Moreover, we did not account for diet and measures of fat deposition, which would have been useful to further understand the lipid data. Additional biomarkers used in cardiovascular risk assessment such as blood glucose and brain natriuretic peptide would have better profiled the CCS cohort. We did not use cardiac strain or strain-rate which could have been useful in the assessments of associations between vascular and cardiac measures. Studies in this regard are ongoing at our institution.

\section{Strengths}

The strengths of the study are; 1) the young CCS cohort, 2) absence of chronic diseases, 3) absence of ongoing medical treatments, and 4) the narrow age-span of the study cohort. The methods used are also widely available and reproducible and future follow up of the study cohort is possible.

\section{REFERENCES}

1. Mertens AC, Liu Q, Neglia JP, Wasilewski K, Leisenring W, Armstrong GT, et al. Cause-specific late mortality among 5-year survivors of childhood cancer: the Childhood Cancer Survivor Study. J Natl Cancer Inst. (2008) 100:1368-79. doi: 10.1093/jnci/djn310

2. Kero AE, Järvelä LS, Arola M, Malila N, Madanat-Harjuoja LM, Matomäki J, et al. Late mortality among 5-year survivors of early onset cancer: a populationbased register study. Int J Cancer. (2015) 136:1655-64. doi: 10.1002/ijc.29135

3. Leerink JM, de Baat EC, Feijen E, Bellersen L, van Dalen EC, Grotenhuis $\mathrm{HB}$, et al. Cardiac disease in childhood cancer survivors: risk prediction,

\section{CONCLUSIONS}

Young Swedish CCS without overt CVD show changes in the cardiovascular system, lipid, and apolipoprotein profiles with potential implications for their CVD risk later in life. Large prospective studies are needed to further assess the course and significance of these changes in clinical CVD.

\section{DATA AVAILABILITY STATEMENT}

The raw data supporting the conclusions of this article will be made available by the authors, without undue reservation.

\section{ETHICS STATEMENT}

The studies involving human participants were reviewed and approved by Etiknämnden Lunds Universitet (DNR 2013/205). The patients/participants provided their written informed consent to participate in this study.

\section{AUTHOR CONTRIBUTIONS}

OB: study design, data collection, and manuscript writing. CW, TW, IØ, and PL: study design and manuscript writing. All authors contributed to the article and approved the submitted version.

\section{FUNDING}

This study was funded by the Swedish Childhood Cancer Fund and the Swedish Heart-Lung Foundation (grant sp2017).

\section{ACKNOWLEDGMENTS}

We are indebted to research nurses Annika Maxedius and Elin Friberg for assistance with data collection (anthropometrics, blood pressure, ECG, questionnaire, and PAT-RHI), Philippe Wagner, biostatistician, for assistance with statistical analyses. Biomedical analyst Judith Huynh for help with interreliability measurements. Professor Peter Nilsson, MD, for his support, knowledge, discussions, and manuscript revision. We are grateful to the Swedish Childhood Cancer Fund and the Swedish HeartLung Foundation for the generous financial support to conduct this study.

prevention, and surveillance: JACC CardioOncology State-of-the-Art Review. JACC CardioOncology. (2020) 2:363-78. doi: 10.1016/j.jaccao.2020.08.006

4. Krawczuk-Rybak M, Tomczuk-Ostapczuk M, Panasiuk A, Goscik E. Carotid intima-media thickness in young survivors of childhood cancer. J Med Imaging Radiat Oncol. (2017) 61:85-92. doi: 10.1111/1754-9485.12510

5. Jenei Z, Bárdi E, Magyar MT, Horváth A, Paragh G, Kiss C. Anthracycline causes impaired vascular endothelial function and aortic stiffness in long term survivors of childhood cancer. Pathol Oncol Res. (2013) 19:37583. doi: 10.1007/s12253-012-9589-6

6. Giordano P, Muggeo P, Delvecchio M, Carbonara S, Romano A, Altomare M, et al. Endothelial dysfunction and cardiovascular risk factors in childhood 
acute lymphoblastic leukemia survivors. Int J Cardiol. (2017) 228:6217. doi: $10.1016 /$ j.ijcard.2016.11.025

7. Dengel DR, Kelly AS, Zhang L, Hodges JS, Baker KS, Steinberger J. Signs of early sub-clinical atherosclerosis in childhood cancer survivors. Pediatr Blood Cancer. (2014) 61:532-7. doi: 10.1002/pbc.24829

8. Brouwer CA, Postma A, Hooimeijer HL, Smit AJ, Vonk JM, van Roon AM, et al. Endothelial damage in long-term survivors of childhood cancer. J Clin Oncol. (2013) 31:3906-13. doi: 10.1200/JCO.2012.46.6086

9. Sadurska E, Brodzisz A, Zaucha-Prazmo A, Kowalczyk J. The estimation of intima-media thickness and cardiovascular risk factors in young survivors of childhood cancer. J Pediatr Hematol Oncol. (2016) 38:54954. doi: 10.1097/MPH.0000000000000513

10. Polak JF, Pencina MJ, Pencina KM, O'Donnell CJ, Wolf PA, D'Agostino RB. Carotid-wall intima-media thickness and cardiovascular events. N Engl J Med. (2011) 365:213-21. doi: 10.1056/NEJMoa1012592

11. Yang EY, Chambless L, Sharrett AR, Virani SS, Liu X, Tang $\mathrm{Z}$, et al. Carotid arterial wall characteristics are associated with incident ischemic stroke but not coronary heart disease in the Atherosclerosis Risk in Communities (ARIC) study. Stroke. (2012) 43:103-8. doi: 10.1161/STROKEAHA.111.626200

12. Zaletel LZ, Popit M, Zaletel M. Is carotid stiffness a possible surrogate for stroke in long-term survivors of childhood cancer after neck radiotherapy? Radiol Oncol. (2018) 52:136-42. doi: 10.2478/raon-2018-0006

13. Palombo C, Kozakova M. Arterial stiffness, atherosclerosis and cardiovascular risk: Pathophysiologic mechanisms and emerging clinical indications. Vascul Pharmacol. (2016) 77:1-7. doi: 10.1016/j.vph.2015.11.083

14. Chueh HW, Yoo JH. Metabolic syndrome induced by anticancer treatment in childhood cancer survivors. Ann Pediatr Endocrinol Metab. (2017) 22:829. doi: 10.6065/apem.2017.22.2.82

15. Felicetti F, Fortunati N, Brignardello E. Cancer survivors: An expanding population with an increased cardiometabolic risk. Diabetes Res Clin Pract. (2018) 143:432-42. doi: 10.1016/j.diabres.2018.02.016

16. Wiebe T, Hjorth L, Marotta Kelly M, Linge HM, Garwicz S. A population based pediatric oncology registry in Southern Sweden: the BORISS registry. Eur J Epidemiol. (2018) 33:1125-1129. doi: 10.1007/s10654-018-0437-1

17. Children's Oncology Group. Guidelines for Long Term Follow Up for Survivors of Childhood, Adolescent and Young Adult Cancer Version 3. (2008). Available online at: $\mathrm{http}: / / \mathrm{www}$.survivorshipguidelines.org/pdf/ltfuguidelines. pdf (accessed June 6, 2020).

18. Odermarsky M, Nilsson A, Lernmark A, Sjöblad S, Liuba P. Atherogenic vascular and lipid phenotypes in young patients with Type 1 diabetes are associated with diabetes high-risk HLA genotype. Am J Physiol. (2007) 293:H3175-9. doi: 10.1152/ajpheart.00795.2007

19. Grubb A. Non-invasive estimation of glomerular filtration rate (GFR). The Lund model: Simultaneous use of cystatin C- and creatinine-based GFRprediction equations, clinical data and an internal quality check. Scand J Clin Lab Invest. (2010) 70:65-70. doi: 10.3109/00365511003642535

20. Stein JH, Korcarz CE, Hurst RT, Lonn E, Kendall CB, Mohler ER, et al. American society of echocardiography carotid intima-media thickness task force. Use of carotid ultrasound to identify subclinical vascular disease and evaluate cardiovascular disease risk: a consensus statement from the American Society of echocardiography carotid intima-media thickness task force. Endorsed by the society for vascular medicine. J Am Soc Echocardiogr. (2008) 21:2. doi: 10.1016/j.echo.2007.11.011

21. Lang RM, Badano LP, Mor-Avi V, Afilalo J, Armstrong A, Ernande L, et al. Recommendations for cardiac chamber quantification by echocardiography in adults: an update from the American Society of Echocardiography and the European Association of Cardiovascular Imaging. J Am Soc Echocardiography. (2015) 1:1. doi: 10.1016/j.echo.2014.10.003

22. Hirai T, Sasayama S, Kawasaki T, Yagi S. Stiffness of systemic arteries in patients with myocardial infarction. A noninvasive method to predict severity of coronary atherosclerosis. Circulation. (1989) 80:78-86. doi: 10.1161/01.CIR.80.1.78

23. Juonala M, Järvisalo MJ, Mäki-Torkko N, Kähönen M, Viikari JS, Raitakari OT. Risk factors identified in childhood and decreased carotid artery elasticity in adulthood: the Cardiovascular Risk in Young Finns Study. Circulation. (2005) 112:1486-93. doi: 10.1161/CIRCULATIONAHA.104. 502161
24. Bonetti PO, Pumper GM, Higano ST, Holmes DR, Kuvin JT, Lerman A. Noninvasive identification of patients with early coronary atherosclerosis by assessment of digital reactive hyperemia. J Am Coll Cardiol. (2004) 44:213741. doi: $10.1016 /$ j.jacc.2004.08.062

25. Young A, Garcia M, Sullivan SM, Liu C, Moazzami K, Ko YA, et al. Impaired peripheral microvascular function and risk of major adverse cardiovascular events in patients with coronary artery disease. Arteriosclerosis Thrombosis Vasc Biol. (2021) 41:5. doi: 10.1161/ATVBAHA.121.316083

26. McQueen MJ, Hawken S, Wang X, Ounpuu S, Sniderman A, Probstfield J, et al. Lipids, lipoproteins, and apolipoproteins as risk markers of myocardial infarction in 52 countries (the INTERHEART study): a case-control study. Lancet. (2008) 19:372. doi: 10.1016/S0140-6736(08)61076-4

27. Georgiopoulos G, Aimo A, Barison A, Magkas N, Emdin M, Masci PG. Imaging predictors of incident heart failure: a systematic review and metaanalysis. J Cardiov Med. (2021) 22:5. doi: 10.2459/JCM.0000000000001133

28. Yuan C, Wang J, Ying M. Predictive value of carotid distensibility coefficient for cardiovascular diseases and all-cause mortality: a meta-analysis. PLoS ONE. (2016) 5:11:e0152799. doi: 10.1371/journal.pone.0152799

29. van Sloten TT, Schram MT, van den Hurk K, Dekker JM, Nijpels G, Henry RM, et al. Local stiffness of the carotid and femoral artery is associated with incident cardiovascular events and all-cause mortality: the Hoorn study. J Am Coll Cardiol. (2014) 63:1739-47. doi: 10.1016/j.jacc.2013.12.041

30. Arnold N, Merzenich H, Wingerter A, Schulz A, Schneider A, Prochaska $\mathrm{JH}$ et al. Promotion of arterial stiffness by childhood cancer and its characteristics in adult long-term survivors. JAHA. (2021) 10:15609. doi: 10.1161/JAHA.119.015609

31. Sundholm J, Suominen A, Sarkola T, Jahnukainen K. Early arterial intimal thickening and plaque is related with treatment regime and cardiovascular disease risk factors in young adults following childhood hematopoietic stem cell transplantation. J Clin Med. (2020) 9:7. doi: 10.3390/jcm9072208

32. Mottram PM, Haluska BA, Leano R, Carlier S, Case C, Marwick TH. Relation of arterial stiffness to diastolic dysfunction in hypertensive heart disease. Heart. (2005) 91:12. doi: 10.1136/hrt.2004.046805

33. Bansal N, Blanco JG, Sharma UC, Pokharel S, Shisler S, Lipshultz SE. Cardiovascular diseases in survivors of childhood cancer. Cancer Metastasis Rev. (2020) 39:1. doi: 10.1007/s10555-020-09859-w

34. Zhang J, Chowienczyk PJ, Spector TD, Jiang B. Relation of arterial stiffness to left ventricular structure and function in healthy women. Cardiovasc Ultrasound. (2018) 16:21. doi: 10.1186/s12947-018-0139-6

35. Kim HL, Lim WH, Seo JB, Chung WY, Kim SH, Kim MA, et al. Association between arterial stiffness and left ventricular diastolic function in relation to gender and age. Medicine. (2017) 96:e5783. doi: 10.1097/MD.0000000000005783

36. Soultati A, Mountzios G, Avgerinou C, Papaxoinis G, Pectasides D, Dimopoulos MA, et al. Endothelial vascular toxicity from chemotherapeutic agents: preclinical evidence and clinical implications. Cancer Treat Rev. (2012) 38:2. doi: 10.1016/j.ctrv.2011.09.002

37. Uhm JS, Chung WB, Yoon JS, Oh YS, Youn HJ. Effects of adriamycin and candesartan on the collagen and elastin of the aorta in rats. Clin Hypertens. (2014) 25:20. doi: 10.1186/2056-5909-1-2

38. Ping Z, Peng Y, Lang H, Xinyong C, Zhiyi Z, Xiaocheng et al. Oxidative stress in radiation-induced cardiotoxicity. Oxidative Med Cell Longevity. (2020) 2020:3579143. doi: 10.1155/2020/3579143

39. Wang $Y$, Boerma M, Zhou D. Ionizing radiation-induced endothelial cell senescence and cardiovascular diseases. Radiation Res. (2016) 186:2 doi: 10.1667/RR14445.1

40. Nikitaki Z, Mavragani IV, Laskaratou DA, Gika V, Moskvin VP, Theofilatos $\mathrm{K}$, et al. Systemic mechanisms and effects of ionizing radiation: A new "old" paradigm of how the bystanders and distant can become the players. Seminars Cancer Biol. (2016) 37-8:77-95. doi: 10.1016/j.semcancer.2016.02.002

41. Russi EG, Raber-Durlacher JE, Sonis ST. Local and systemic pathogenesis and consequences of regimen-induced inflammatory responses in patients with head and neck cancer receiving chemoradiation. Media Inflamm. (2014) 2014:518261. doi: 10.1155/2014/518261

42. van Nimwegen FA, Schaapveld M, Janus CP, Krol AD, Petersen EJ, Raemaekers JM, et al. Cardiovascular disease after Hodgkin lymphoma treatment: 40-year disease risk. JAMA Internal Med. (2015) 175:6 doi: 10.1001/jamainternmed.2015.1180 
43. Lipshultz SE, Landy DC, Lopez-Mitnik G, Lipsitz SR, Hinkle AS, Constine LS, et al. Cardiovascular status of childhood cancer survivors exposed and unexposed to cardiotoxic therapy. J Clin Oncol. (2012) 30:10. doi: 10.1200/JCO.2010.33.7907

44. Armenian SH, Hudson MM, Mulder RL, Chen MH, Constine LS, Dwyer $\mathrm{M}$, et al. Recommendations for cardiomyopathy surveillance for survivors of childhood cancer: a report from the international late effects of childhood cancer guideline harmonization group. Lancet Oncol. (2015) 16:3. doi: 10.1016/S1470-2045(14)70409-7

45. Aleman BM, van den Belt-Dusebout AW, Klokman WJ, Van't Veer MB, Bartelink $\mathrm{H}$, van Leeuwen FE. Long-term cause-specific mortality of patients treated for Hodgkin's disease. J Clin Oncol. (2003) 21:18. doi: 10.1200/JCO.2003.07.131

46. Rubinshtein R, Kuvin JT, Soffler M, Lennon RJ, Lavi S, Nelson RE, et al. Assessment of endothelial function by non-invasive peripheral arterial tonometry predicts late cardiovascular adverse events. Eur Heart J. (2010) 31:9. doi: 10.1093/eurheartj/ehq010

47. Ruble K, Davis CL, Han HR. Endothelial health in childhood acute lymphoid leukemia survivors: pilot evaluation with peripheral artery tonometry. J Pediatr Hematol Oncol. (2015) 37:2. doi: 10.1097/MPH.0000000000 000122

48. Zelcer S, Chen B, Mangel J, Vujovic O, Thiessen-Philbrook HR, Reider M, et al. Impaired vascular function in asymptomatic young adult survivors of Hodgkin Lymphoma following mediastinal radiation. J Cancer Surviv. (2010) 4:138. doi: 10.1007/s11764-010-0138-6

49. Bruno RM, Gori T, Ghiadoni L. Endothelial function testing and cardiovascular disease: focus on peripheral arterial tonometry. Vasc Health Risk Manag. (2014) 10:577-84. doi: 10.2147/VHRM.S44471

50. Smith JD. Apolipoprotein A-I and its mimetics for the treatment of atherosclerosis. Curr Opin Investig Drugs. (2010) 11:989-96.

51. Kluck G, Durham KK, Yoo JA, Trigatti BL. High density lipoprotein and its precursor protein apolipoprotein A1 as potential therapeutics to prevent anthracycline associated cardiotoxicity. Front Cardiovasc Med. (2020) 7:65. doi: $10.3389 /$ fcvm.2020.00065

52. Sniderman AD, Thanassoulis G, Glavinovic T, Navar AM, Pencina M, Catapano A, et al. Apolipoprotein B particles and cardiovascular disease: a narrative review. JAMA Cardiol. (2019) 4:12. doi: 10.1001/jamacardio.2019.3780

53. Follin C, Erfurth EM. Long-term effect of cranial radiotherapy on pituitaryhypothalamus area in childhood acute lymphoblastic leukemia survivors. Curr Treat Options Oncol. (2016) 17:50. doi: 10.1007/s11864-016-0426-0

54. Sklar CA, Antal Z, Chemaitilly W, Cohen LE, Follin C, Meacham LR, et al. Hypothalamic-pituitary and growth disorders in survivors of childhood cancer: an endocrine society clinical practice guideline. J Clin Endocrinol Metab. (2018) 103:2761-84. doi: 10.1210/jc.2018-01175

Conflict of Interest: The authors declare that the research was conducted in the absence of any commercial or financial relationships that could be construed as a potential conflict of interest.

Publisher's Note: All claims expressed in this article are solely those of the authors and do not necessarily represent those of their affiliated organizations, or those of the publisher, the editors and the reviewers. Any product that may be evaluated in this article, or claim that may be made by its manufacturer, is not guaranteed or endorsed by the publisher.

Copyright (c) 2021 Broberg, Øra, Wiebe, Weismann and Liuba. This is an open-access article distributed under the terms of the Creative Commons Attribution License (CC $B Y)$. The use, distribution or reproduction in other forums is permitted, provided the original author(s) and the copyright owner(s) are credited and that the original publication in this journal is cited, in accordance with accepted academic practice. No use, distribution or reproduction is permitted which does not comply with these terms. 